\title{
Stress corrosion of organosilicate glass films in aqueous environments: Role of pH
}

\author{
F. Iacopi ${ }^{\text {a) }}$ \\ Inter-university Micro-Electronics Center (IMEC), Kapeldreef 75, B-3001 Leuven, Belgium \\ C. Elia \\ Inter-university Micro-Electronics Center (IMEC), Kapeldreef 75, B-3001 Leuven, Belgium; and \\ Istituto Universitario degli Studi Superiori di Pavia, Pavia, Italy \\ T. Fournier \\ Inter-university Micro-Electronics Center (IMEC), Kapeldreef 75, B-3001 Leuven, Belgium; and \\ Génie des Matériaux, Université Aix-Marseille III, Marseille, France \\ F. Sinapi and Y. Travaly \\ Inter-university Micro-Electronics Center (IMEC), Kapeldreef 75, B-3001 Leuven, Belgium
}

(Received 27 May 2007; accepted 2 January 2008)

\begin{abstract}
Subcritical cracking of thin glass films caused by stress-corrosion phenomena cannot be neglected when it comes to application and manufacturing processes that involve exposure to aqueous environments. A protocol is introduced to allow for a quantitative study of stress corrosion through channel cracking experiments. By this method, an exponential dependence of the crack propagation rate on the $\mathrm{pH}$ of the aqueous environment is revealed. Therefore, this behavior should be accounted for through the use of an appropriate pre-exponential factor in the expression of channel cracking rate. This factor should reflect the reduced crack resistance of the glass film caused by the weakening of the silica bonds behind the crack tip in the aqueous environment. A direct comparison between commercial slurries and reference solutions confirms that the crack resistance is a function of the $\mathrm{pH}$ of the ambient.
\end{abstract}

\section{INTRODUCTION}

In recent years the microelectronics community has focused its attention on alternative materials to comply with the requirements given by the dramatic downscaling of feature sizes. One example is organosilicate glasses (OSGs) or carbon-doped silicon oxides, known as lowdielectric-constant ("low- $k$ ") materials. ${ }^{1}$

The use of these dielectrics as a replacement for $\mathrm{SiO}_{2}$ in interconnects technology reduces the dynamic power consumption and parasitic resistance-capacitance (RC) delay. ${ }^{1}$ The silica bond chains of organosilicate glasses are terminated by $-\mathrm{CH}_{3}$ hydrophobic groups. This leads to a net decrease in density and polarizability of the silica matrix and thus to a lower dielectric constant than $\mathrm{SiO}_{2}$. To decrease the dielectric constant even further, large amounts of porosity (up to $50 \%$ of the volume) can be incorporated into the OSG films. The introduction of porosity deteriorates the mechanical properties of the organosilicate films. The Young's modulus and the hard-

\footnotetext{
a) Address all correspondence to this author.

e-mail: francesca.iacopi@imec.be

DOI: $10.1557 / J M R .2008 .0108$
}

ness of such films decrease abruptly as film porosity increases. ${ }^{2}$ Cohesive strength of such films is also dramatically affected by the reduction of bond density.

The worsening of mechanical properties of porous OSG films, combined with intrinsic stresses generated during the growth of the films and with stresses generated by mechanical loads during interconnects manufacturing processes ${ }^{2,3}$ such as thermal cycles or chemicalmechanical polishing processes, ${ }^{4}$ leads to an increased probability of generating film fracture. A typical failure mode of these materials during device fabrication is channel cracking. The driving force $(G)$ for channel cracking $^{5-7}$ is expressed as the elastic energy reduction associated with the increase of the crack length by the relation (1)

$$
G=Z \frac{\pi}{2} \frac{\left(1-v_{\mathrm{f}}^{2}\right) \sigma^{2} a}{E_{\mathrm{f}}},
$$

where $Z$ is a constant that depends on the elastic mismatch between film and substrate and on geometric constrains, $\nu_{\mathrm{f}}$ is the Poisson's ratio of the film, $\sigma$ is the stress of the film, $E_{\mathrm{f}}$ is the Young's modulus of the film, and $a$ is the thickness of the crack. Note that for a compliant 
film on a stiff substrate (i.e., OSG film on a silicon substrate), the crack depth $a$ can be set equal to the film thickness $h$.

When the crack grows, energy is spent to break the bonds of the material. The crack resistance $R$ is defined as the energy necessary for a crack to grow. Only if the driving force for the crack to propagate is higher than the crack resistance $(G>R)$ the crack can propagate. The relationship between crack velocity and the crack driving force is expressed by relation (2)

$$
V=V_{0} e^{G / 2 n K T}=V_{0} e^{Z / 2 n K T\left\{\left[\pi\left(1-\nu_{\mathrm{f}}^{2}\right) \sigma^{2} h\right] / 2 E_{\mathrm{f}}\right\}},
$$

where $K$ is the Boltzmann constant, $T$ is the absolute temperature, $n$ is the bond density, $h$ is the film thickness, and $V_{0}$ is a reference velocity. ${ }^{8}$ Relation (2) highlights the dependence of the crack velocity on the characteristics of the material. However, the dependence of the crack speed on the environments in which the crack grows is not immediately explicit in the relation (2). Much of the recent work on thin-glass films deals with the dependence on the environment by using the preexponential factor $V_{0}$ as a simple fitting parameter ${ }^{7}$ and not as a function of species concentration. A correct description of $V_{0}$ is critical, as environmental conditions different from the ambient environment can lead to subcritical crack propagation and thus to premature failure due to stress corrosion, as demonstrated for bulk glasses. ${ }^{9}$

Guyer and Dauskardt ${ }^{10}$ studied in detail the effect of different aqueous solutions on the crack-growth rate of OSG films, using the double cantilever beam method with sandwiched geometry. In their work they reported that acid environments generally inhibit the crack growth compared with deionized water, except for solutions with $\mathrm{H}_{2} \mathrm{O}_{2}$, for which they reported higher cracking rates. They also reported an enhancement of crack-growth rates in basic solutions, explained through stress corrosion of the $\mathrm{Si}-\mathrm{O}$ strained bonds at the crack tip mediated by the $\mathrm{OH}^{-}$anions. Somewhat conflicting results were reported by Jacques et al., ${ }^{11}$ by monitoring channel cracking. No clear dependence of the crack velocity on the $\mathrm{pH}$ of the solution was observed in their study, although they did report higher crack propagation rates in water compared with dry ambient. Studies of channel cracking generally led to more uncertainty in the quantitative evaluation of the fracture properties of the films compared with studies performed with double cantilever beam. Two main reasons are that the driving force for channel cracking is not variable but intrinsically defined by the materials and geometry used, and that this method relies on the optical observation and evaluation of the crack lengths.

Therefore, double cantilever beam is a preferred technique for accurate studies of the fundamental fracture properties of thin film. However, channel cracking is a failure mode that is technologically relevant. A better understanding of how aqueous solutions influence the channel crack rate is important for predicting a safe window for the manufacturability of interconnects with porous OSG dielectrics, as the films are often exposed to wet processing environments.

In this work we study crack propagation by monitoring channel cracking as Jacques et al., ${ }^{11}$ but we confirm the main results obtained by Guyer and Dauskardt. ${ }^{10}$ We report an exponential dependence of the crack-growth rate on the $\mathrm{pH}$ of the solution, and we attempt to explain this dependence on the pre-exponential factor $V_{0}$ in (2), accounting for the reduced-film resistance to cracking in aqueous environments. Hence, we show that accurate monitoring of channel cracking yields results of general validity, provided some critical precautions are taken.

\section{EXPERIMENTAL}

We selected three different OSG film types on Si substrates, characterized by different values of porosity, dielectric constant $(k)$, Young's modulus, and residual stress. Film thickness was measured by conventional ellipsometry, while film porosity and pore size was determined with ellipsometric porosimetry using toluene as solvent. ${ }^{12}$ The dielectric constant of the OSG films was obtained from capacitance measurements at $100 \mathrm{kHz}$ on planar capacitors structures. ${ }^{1}$ Nanoindentation with a Berkovich tip and surface acoustic wave measurements ${ }^{13}$ on 1- $\mu \mathrm{m}$-thick films were performed to extract the value of Young's modulus for each of OSG material type. Fitting of the dispersion of the acoustic modes measured on $1-\mu \mathrm{m}$ films with surface acoustic waves indicated that a Poisson's ratio of about 0.2 is a good assumption for the studied films. Dispersion for acoustic wavelengths from 4 to $10 \mu \mathrm{m}$ was measured, using film thickness and density as input parameters. The Young's modulus values retrieved from such measurements were in good agreement with those measured by nanoindentation. Residual film stress was obtained from the measurement of wafer warpage on 300-mm Si wafers. Table I summarizes the main film properties and the driving

TABLE I. Values of Young's modulus $(E)$, tensile stress $(\sigma)$, thickness $(h)$, and driving force $(G)$ for crack propagation for each OSG material sample.

\begin{tabular}{lccrccc}
\hline \hline & $E(\mathrm{GPa})$ & $\sigma(\mathrm{GPa})$ & $h(\mathrm{~nm})$ & $G\left(\mathrm{~J} / \mathrm{m}^{2}\right)$ & $k$ & Porosity $(\%)$ \\
\hline OSG1 & 4.5 & 0.1 & 1400 & 3.85 & 2.4 & 30 \\
& & & 1200 & 3.30 & & \\
OSG2 & 7.4 & 0.05 & 2400 & 2.20 & & \\
& & & 2200 & 0.92 & & \\
& & & 2000 & 0.84 & & \\
& & & 1800 & 0.75 & & 2.00 \\
OSG3 & 7.5 & 0.054 & 1000 & 0.48 & 2.9 & 23 \\
& & 0.053 & 800 & 0.37 & & \\
\hline \hline
\end{tabular}


forces $(G)$ for crack propagation calculated, for each thickness, by relation (1).

The $Z$ value for our experimental conditions is calculated based on the analysis from Beuth ${ }^{14}$ and is $Z=0.82$. All the selected materials are microporous, i.e., with pore size smaller than $2 \mathrm{~nm}$. The porosity of the films varies between $23 \%$ and $30 \%$. Note that the thicknesses $(h)$ were chosen such to cover different ranges of nominal driving forces. The OSG1 film samples exhibit the highest values of $G$, OSG2 samples possess intermediate values, while OSG3 films have the smallest $G$ values.

Several solutions are used to study effects on crack growth from wet environments (Table II). Some of the solutions are technologically relevant, i.e., slurries used for chemical mechanical polishing of copper and metal barrier layers, here referred to as S1, S2, and S3. Other solutions were chosen to cover the complete $\mathrm{pH}$ spectrum in this experiment or to have reference solutions with the same $\mathrm{pH}$ as the commercial slurries. Channel cracking was studied in a controlled environment at a temperature of $20 \pm 1^{\circ} \mathrm{C}$. The samples used in the experiment consisted of OSG films onto $\mathrm{Si}$ substrates with square $2 \times 2 \mathrm{~cm}^{2}$ size. The chosen OSG materials are commercially available low-dielectric constant materials. The OSG1 was spin-on cast, whereas OSG2 and OSG3 were deposited via chemical vapor deposition. On the samples a scratch was made with a sharp diamond scribe. Cracks on the sample were induced by a scratch with depth larger than the thickness of the film. The film samples were immersed in the different solutions as in Table II. The solutions S1, S2, and S3 were mixtures of commercially available slurries with variable content of colloidal silica, mixed with hydrogen peroxide: $\mathrm{S} 1$ is an acidic copper slurry with $9 \% \mathrm{H}_{2} \mathrm{O}_{2}, \mathrm{~S} 2$ is an acidic selective barrier slurry with $0.26 \% \mathrm{H}_{2} \mathrm{O}_{2}, \mathrm{~S} 3$ is a caustic nonselective slurry with $0.2 \% \mathrm{H}_{2} \mathrm{O}_{2}$. The buffer solution is produced by Merck and is composed by boric acid, potassium chloride, and sodium hydroxide. Crack length was measured at regular intervals of time using an optical microscope. The crack-growth rate was defined as the ratio between the increase of crack length and the time interval elapsed between the two measurements. To obtain statistically significant results, all cracks nucleated on each scratch were monitored. Every crack trace was followed until it reached the edge of the sample.

Nanoindentation measurements were repeated on samples without crack initiation after 1 week storage in the different aqueous solutions used in this work, indi-

TABLE II. Aqueous solutions used for the environmental studies.

\begin{tabular}{cccccccc}
\hline \hline & $\begin{array}{c}\mathrm{H}_{2} \mathrm{O}_{2} 30 \%+ \\
\mathrm{HNO}_{3} 0.02 \%\end{array}$ & $\mathrm{~S} 3$ & $\mathrm{~S} 1$ & $\begin{array}{c}\mathrm{H}_{2} \mathrm{O}_{2} \\
10 \%\end{array}$ & $\mathrm{H}_{2} \mathrm{O}$ & $\mathrm{S} 3$ & Buffer \\
\hline $\mathrm{pH}$ & 2.5 & 2.5 & 3.4 & 4.5 & 6.6 & 8.7 & 10.0 \\
\hline \hline
\end{tabular}

cating no significant change from the Young's modulus values of the films in Table I. Film residual stress, measured on full Si wafer after a rinse and dry procedure, was found stable for integer films at least for up to $1 \mathrm{~h}$ immersion in either deionized water or in the slurries for chemical-mechanical polishing.

\section{RESULTS AND DISCUSSION}

As highlighted in Table I, the chosen samples cover a large range of $G$ values. We show that a first precaution for accurate measurements of channel cracking consists of the appropriate choice of the $G$ range and thus the choice of suitable thicknesses for a given material type. For this purpose, we chose the highly basic S3 solution, expected to be the most aggressive slurry. In Fig. 1(a) a microscope image of the $1200 \mathrm{~nm}$ thick OSG1 sample is reported after $2 \mathrm{~h}$ of dipping in S3. The density of cracks is too high to allow a reliable measurement of the propagation rate. On the other extreme, the OSG3 material does not present propagation of cracks from the initial scratch after one month storage in S3 [Figs. 1(c) and 1(d)]. The OSG2 samples, characterized by intermediate $G$ values, exhibit the most suitable behavior: a small density of cracks departing from the initial scratch [Fig. $1(b)$, enabling the study of crack propagation for a length of several $\mu \mathrm{m}$. It follows that for OSG films with porosity around $25 \%$, a nominal $G$ value roughly around $1 \mathrm{~J} / \mathrm{m}^{2}$ is recommended to enable channel cracking studies in a broad $\mathrm{pH}$ spectrum. For this reason, we focused on OSG2 samples to study in detail the effect of wet environments on channel cracking.

A second important precaution for ensuring reliable measurements concerns the length threshold for the cracks before reaching a steady-state propagation rate according to the expression (2). The initial scratch and the concentration of cracks departing from the scratch location produce a modification of the local stress fields. It is known that the cracks will not propagate in steadystate conditions until their tip is sufficiently far away from the initial scratch (and other propagating cracks) and will only feel the residual uniform in-plane residual stress of the film. In literature only vague indications are given on this threshold length, as being several times the thickness of the films. ${ }^{8}$ Therefore, a systematic study of the behavior of the crack velocity versus crack length is performed to evaluate the magnitude of this threshold. In Fig. 2(a), the data obtained for samples of 2400, 2200, and $2000 \mathrm{~nm}$ dipped in S2 are reported. The figure shows that cracks with lengths below the threshold exhibit lower velocities than at the steady state. This is because the local film stress in the region within the threshold length is partially relaxed by the initial scratch and the neighboring cracks. The plot in Fig. 2(b) confirms that the threshold length scales linearly with film thickness, 

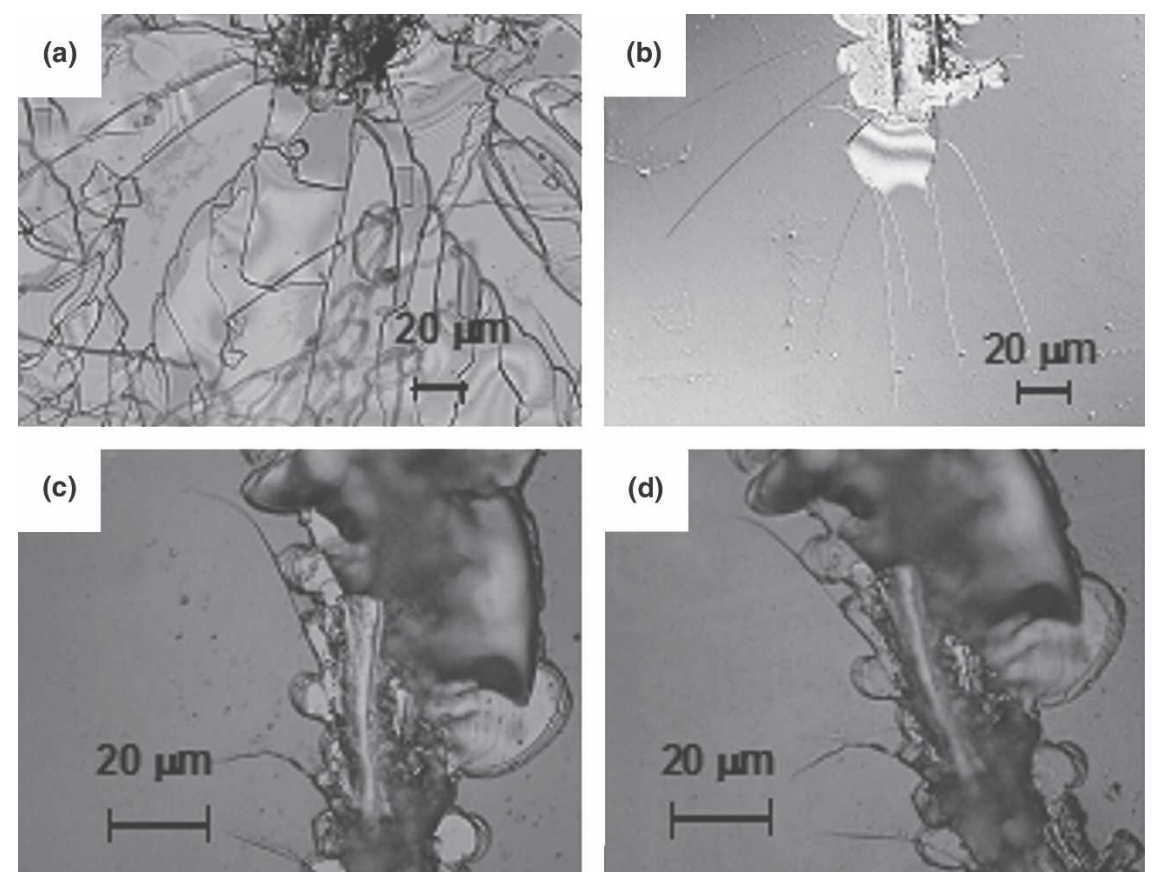

FIG. 1. Different regimes of crack rate are observed upon immersion in the S3 solution, depending on the $G$ values of the samples as reported in Table I. (a) High $G$ value: the $1200 \mathrm{~nm}$ OSG1 film shows destructive cracking after only $2 \mathrm{~h}$ dipping. (b) Intermediate $G$ : the $2200 \mathrm{~nm}$ OSG2 sample offers ideal conditions for measuring crack propagation rate (image taken after about 3 weeks dip time). (c, d) Images of the $1000 \mathrm{~nm}$ OSG3 at an interval of 1 month. The driving force is not sufficient to let cracks propagate.

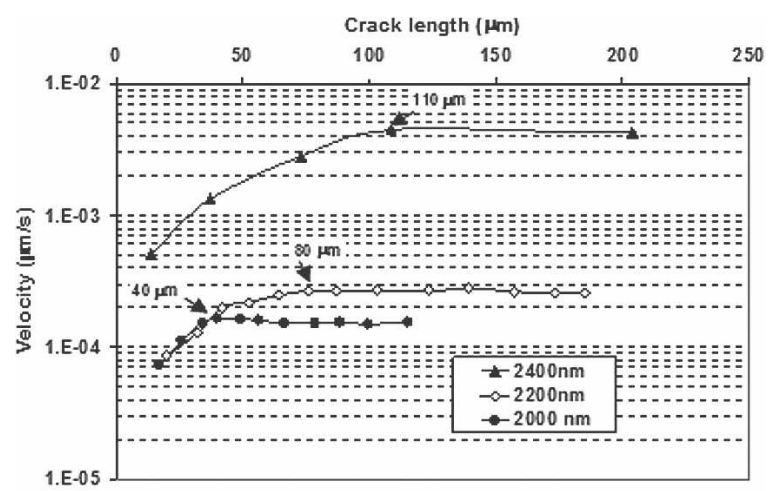

(a)

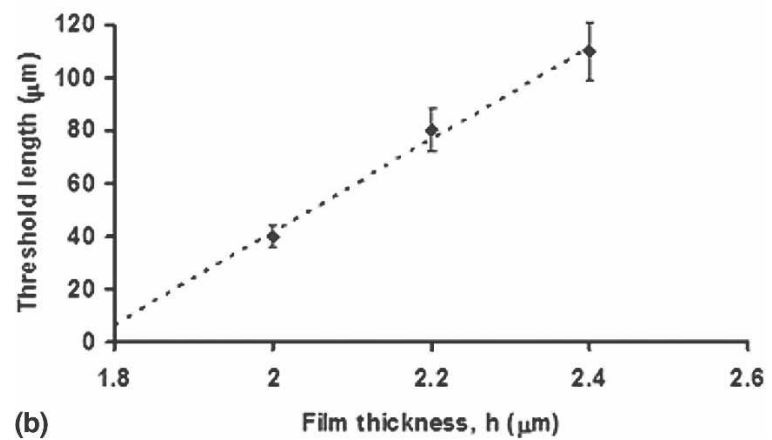

FIG. 2. (a) The threshold lengths needed for the crack to reach a steady-state propagation at constant velocity are compared for OSG2 samples with different thicknesses. (b) The threshold lengths scale linearly with film thickness. which is an expected phenomenon for elastic stress fields in a compliant film constrained onto a stiff substrate. However, the linear regression does not pass through the origin, so a calibration is needed to derive accurately the threshold length for different thicknesses. On the other hand, the threshold is the same regardless of the environment. Only in the case of the buffer solution, with the highest $\mathrm{pH}$, we observed slightly higher threshold values. Figure 2 shows that differences up to 1 order of magnitude can be encountered between the velocity of cracks with length below the threshold and those propagating in steady-state conditions. These results emphasize the importance of an accurate evaluation of the threshold length.

Before investigating the occurrence of stress-corrosion phenomena of OSG films in wet environments, it is interesting to probe exclusively the chemical action of solutions with different $\mathrm{pH}$ onto the films. This is done by evaluating the contact angle of water on the surface of OSG samples dipped into the solutions in Table I for different durations. The initial contact angle with water is $93 \pm 2^{\circ}$ for pristine OSG films. The behavior of contact angle is found to be dependent on the $\mathrm{pH}$ of the used solution. Figure 3 reports the example of the OSG1 samples dipped in slurry solutions $\mathrm{S} 2$ (pH 2.5) and $\mathrm{S} 3 \mathrm{(pH}$ 8.7) for up to about $3 \mathrm{~h}$, indicating that films exposed to basic solutions tend to lose faster their 


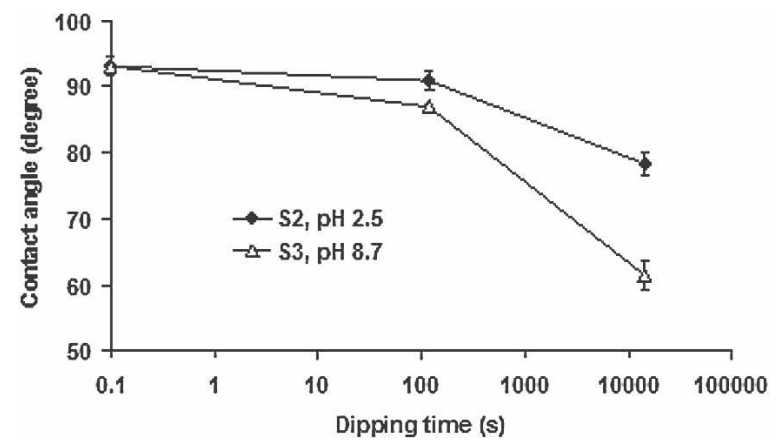

FIG. 3. Behavior of the contact angle with water versus the dipping time in slurries S2 and S3 (OSG1 films).

hydrophobic behavior. This is an indication for a faster chemical attack of the top surface of OSG films in basic environments. Nevertheless, the bulk of the films do not seem to be significantly affected by the exposure to the slurries (or then it does so at a much slower rate), as the stability of elastic modulus and film stress indicates. Based on these results, it is also reasonable to assume that the chemical action of the slurries has, in first approximation, only an influence on discontinuities and pre-existing cracks of the film.

Another simple preliminary experiment confirmed the sensitivity of stress-corrosion phenomena of the OSG films on the $\mathrm{pH}$ of the wet ambient. First, we measured the crack velocity departing from a scratch onto an OSG2 sample in $\mathrm{S} 2$ ( $\mathrm{pH} 2.5)$. When the crack length was above the threshold length, we placed the sample in the highly basic $\mathrm{S} 3$ solution ( $\mathrm{pH}$ 8.7). Figure 4 reports the behavior of the crack velocity for three distinct cracks on the same sample. The steplike increase of the crack velocity corresponds to the change of solution, as indicated by the dashed line. The difference in crack velocity is almost 1 order of magnitude and demonstrates that the $\mathrm{pH}$ is a predominant parameter in the crack propagation.

Figure 5 reports the channel crack velocity data for the 2200-nm-thick OSG2 films immersed in the different

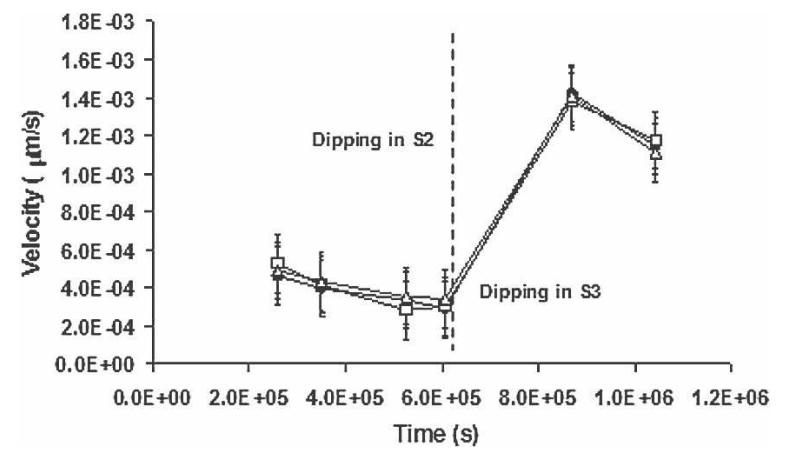

FIG. 4. Crack propagation rate versus dipping time for three different cracks on an OSG2 sample immersed first in the S2 and subsequently in the S3 solution. The abrupt increase corresponds to the change of solution.

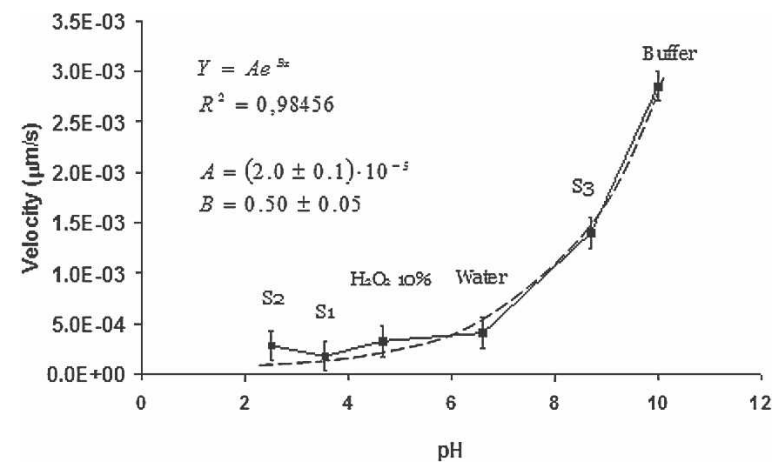

FIG. 5. The crack velocity shows an exponential increase versus the $\mathrm{pH}$ of the wet environment (OSG2 sample, $2200 \mathrm{~nm}$ thick).

solutions: the crack rate shows an exponential increase versus the $\mathrm{pH}$ of the environment. Note that the crack rate in wet environments for OSG films always appears higher than in dry ambient, as films with the same thickness did not exhibit any appreciable crack propagation in ambient atmosphere.

It was also verified that different types of solutions with the same $\mathrm{pH}$ lead to equal crack rates, as demonstrated in Fig. 6. The crack velocity of an OSG2 film in S2 is essentially equal to the propagation rate in a solution of $\mathrm{H}_{2} \mathrm{O}_{2} 30 \%+\mathrm{HNO}_{3} 0.02 \%$ (Table II). Further, we report the absence of an enhancement of crack rate linked to the presence of a higher concentration of hydrogen peroxide. Consequently, we can conclude that the $\mathrm{pH}$ of the solution is the dominating parameter governing stress corrosion of OSG glasses. With the exception of the specific effect of peroxide, the general dependence of the crack velocity on the $\mathrm{pH}$ confirms the findings by Guyer and Dauskardt ${ }^{10}$ with double cantilever beam experiments. The increase of crack rate caused by hydrogen peroxide was reported for driving forces below $2 \mathrm{~J} / \mathrm{m}^{2}$. The discrepancy could be attributed to the different conditions in terms of mechanical loading and solution transport to the crack tip of channel cracking experiments compared with the sandwiched double cantilever beam geometry. This would suggest that our experimental regime is likely to

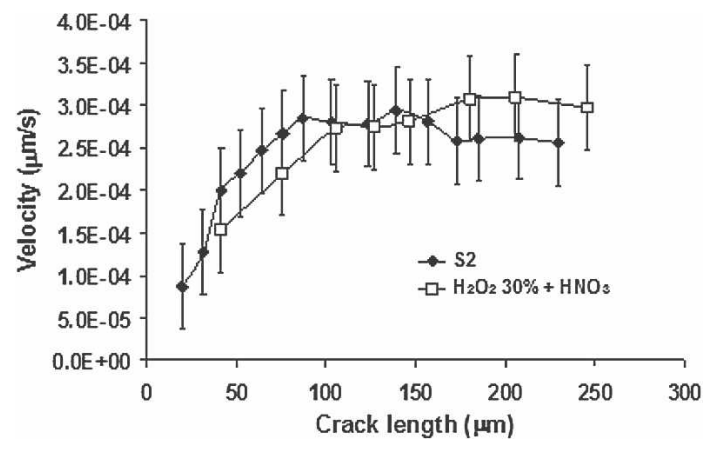

FIG. 6. The crack propagation rate for an OSG2 sample dipped in solutions with the same $\mathrm{pH}$ is found to be equivalent, regardless of the $\mathrm{H}_{2} \mathrm{O}_{2}$ content. 
correspond rather to the reaction-limited region reported by Guyer and Dauskardt ${ }^{10}$ for higher driving forces $(G>$ $2 \mathrm{~J} / \mathrm{m}^{2}$ ). The magnitude of the crack velocities in Fig. 5 $\left(10^{-9}\right.$ to $\left.10^{-10} \mathrm{~m} / \mathrm{s}\right)$ corresponds indeed to the reactionlimited region of the subcritical cracking graphs reported by Guyer and Dauskardt. ${ }^{15}$ This is also in agreement with the work of Cook and Liniger, ${ }^{6}$ who studied channel cracking of OSG films in ambient and water describing it with an absolute reaction-rate model.

The graph of the crack velocity versus the ambient $\mathrm{pH}$ in Fig. 5 shows an exponential behavior, so the fitted curve is of the type

$$
y=A e^{B x},
$$

with $y$ the crack velocity and $x$ the $\mathrm{pH}$ value. The fitting parameters are reported in the figure. This evidence suggests that an explicit dependence on the $\mathrm{pH}$ of the aqueous medium should be introduced in Eq. (2). This cannot be done by using the pre-exponential factor $V_{0}$ as a simple fitting constant. Nevertheless, the observed behavior can be accounted for by making explicit the dependence of $V_{0}$ on the crack resistance $R$, which in turn can be expressed as a function of the concentration of the chemically active species. A qualitative formalism accounting for crack-front reaction kinetics was reported by Lawn, ${ }^{16}$ who expresses the pre-exponential term as

$$
V_{0} \propto \exp (-\alpha R / k T),
$$

with $\alpha$ an activation area factor, $k$ the Boltzmann constant, and $T$ the temperature. The crack resistance $R$ can in turn be expressed as a function of the concentration of the active species (thus the $\mathrm{pH}$, in this case). ${ }^{16}$ This formalism can fully describe our case and is interpreted as the reduction of the crack resistance of the glass films in aqueous environments caused by the weakening of the silica bonds behind the crack tip.

\section{CONCLUSIONS}

Overall, these results confirm a pronounced phenomenon of stress corrosion of OSG materials in aqueous environments. The observed exponential behavior of the crack velocity versus the $\mathrm{pH}$ of the wet media indicates that environmental effects lead to a reduction of the glass film resistance to crack. When OSG films are immersed in aqueous solutions, the silica bonds are weakened in proximity of the crack tip, and consequently the crack resistance of the glass film is lower than in dry ambient.

Therefore, to account for environmental effects on channel cracking in a comprehensive fashion, the preexponential factor $V_{0}$ in the expression for crack velocity should contain the explicit dependence on the crack resistance $R$, which in turn is a function of $\mathrm{pH}$.

We highlight the dramatic enhancement of crack rates of films in highly basic environment. In particular, we identify the $\mathrm{pH}$ of the used solution as the governing parameter for stress corrosion. As do Guyer and Dauskardt, ${ }^{10}$ we also attribute the silica bonds weakening in acidic and neutral solutions to the interaction with $\mathrm{H}_{2} \mathrm{O}$ molecules, as has been shown earlier for bulk glasses. ${ }^{17}$ This effect is less pronounced in acidic ambient. Conversely, we do not observe the anomalous crack behavior in the presence of $\mathrm{H}_{2} \mathrm{O}_{2}$ reported by the same group. The dramatic enhancement of crack rates in basic environments is attributed to the stress corrosion of the $\mathrm{Si}-\mathrm{O}$ bonds at the crack tip assisted by $\mathrm{OH}^{-}$ions. ${ }^{10,18}$ Nevertheless, at the moment we do not exclude a contribution from the more favorable transport kinetics to the crack tip of $\mathrm{OH}^{-}$species, as they have smaller steric hindrance compared with the water molecule and since an accelerated wetting of OSG films by basic solutions was demonstrated in Fig. 3.

Finally, we demonstrate that channel cracking experiments can be a reliable method to study fundamental aspects of fracture as stress corrosion, provided some critical requirements are met. The thickness of the samples should be selected to obtain a driving force allowing for low crack density and yet a reasonable velocity for crack propagation. Second, the threshold crack length for steady-state crack propagation should be carefully evaluated, and only data above this threshold should be considered. We also show that this threshold does not necessarily scale with sample thickness by a fixed factor. Finally, since stress corrosion is found to be extremely sensitive to the $\mathrm{pH}$ of the solution, it is essential to ensure the stability of the $\mathrm{pH}$ of the aqueous environment for the whole duration of the experiment.

The dependence of the subcritical crack rate on the $\mathrm{pH}$ of the wet environments is of general technological relevance, in particular, for interconnects manufacturing, where processing solutions are regularly used for cleaning purposes, and for processes such as chemicalmechanical polishing, where wet environments are used in combination with the application of a mechanical force. In this case, an appropriate choice of the $\mathrm{pH}$ of the process medium is essential to avoid subcritical fracture.

\section{REFERENCES}

1. K. Maex, M.R. Baklanov, D. Shamiryan, F. Iacopi, S.H. Brongersma, and Z.S. Yanovitskaya: Low-dielectric constant materials for microelectronics. J. Appl. Phys. 93, 8793 (2003).

2. F. Iacopi, S.H. Brongersma, B. Vandevelde, M. O'Toole, D. Degryse, Y. Travaly, and K. Maex: Challenges for structural stability of ultra-low-k-based interconnects. Microelectron. Eng. 75(1), 54 (2004).

3. R.J.O.M. Hoofman, V.H. Nguyen, V. Arnal, M. Broekhaart, L.G. Gosset, W.F.A. Besling, M. Fayolle, and F. Iacopi: Integration of low-k dielectric films in damascene processes, in Dielectrics for Microelectronics, edited by M. Baklanov, K. Maex, and 
M. Green (Wiley \& Sons, Chichester, UK, 2007), Chap. 5, pp. 238-239.

4. R.K. Singh and R. Bajaj: Advances in chemical-mechanical planarization. Mater. Res. Soc. Bull. 27(10), 743 (2002).

5. R.F. Cook and Z. Suo: Mechanisms active during fracture under constraint. Mater. Soc. Bull. 27, 45 (2002).

6. R.F. Cook and E.G. Liniger: Stress-corrosion cracking of lowdielectric constant spin-on-glass thin films. J. Electrochem. Soc. 146(12), 4439 (1999).

7. T.Y. Tsui, A.J. Griffin, R. Fields, J.M. Jacques, A. McKerrow, and J.J. Vlassak: The effect of elastic modulus on channel crack propagation in organosilicate glass films. Thin Solid Films 515(4), 2257 (2006).

8. T.Y. Tsui, A.J. McKerrow, and J.J. Vlassak: Constraints effects on thin film channel cracking behavior. J. Mater. Res. 20(9), 2266 (2005).

9. S.M. Wiederhorn and H. Johnson: Influence of sodium-hydrogen ion-exchange on crack propagation in soda-lime silicate glass. J. Am. Ceram. Soc. 56(2), 108 (1973).

10. E.P. Guyer and R.H. Dauskardt: Fracture of nanoporous thin-film glasses. Nat. Mater. 3, 53 (2004).

11. J.M. Jacques, T.Y. Tsui, A.J. McKerrow, and R. Kraft: Environmental effects on crack characteristics for OSG materials, in Thin Films-Stresses and Mechanical Properties XI, edited by T.E. Buchheit, A.M. Minor, R. Spolenak, and K. Takashima
(Mater. Res. Soc. Symp. Proc. 875, Warrendale, PA, 2005), O10.6.1.

12. M.R. Baklanov, K.P. Mogilnikov, V.G. Polovinkin, and F.N. Dultsev: Determination of pore-size distribution in thin films by ellipsometric porosimetry. J. Vac. Sci. Technol., B 18(3), 1385 (2000).

13. F. Iacopi, Y. Travaly, M. Van Hove, A.M. Jonas, J.M. MolinaAldareguia, M.R. Elizalde, and I. Ocaña: Extent of plasma damage to porous organosilicate films characterized with nanoindentation, x-ray reflectivity and surface acoustic waves. J. Mater. Res. 21(12), 3161 (2006).

14. J.L. Beuth, Jr.: Cracking of thin bonded films in residual tension. Int. J. Solids Struct. 29, 1657 (1992).

15. E. Guyer and R.H. Dauskardt: Effect of solution $\mathrm{pH}$ on the accelerated cracking of nanoporous thin-film glasses. J. Mater. Res. 20(3), 680 (2005).

16. B. Lawn: Ch. 5, Chemical processes in crack propagation: Kinetic fracture, in Fracture of Brittle Solids, 2nd ed. (Cambridge University Press, Cambridge, UK, 1993), pp.130-131.

17. S.M. Wiederhorn and H. Johnson: Effect of aqueous-solutions on crack-propagation of glass. Am. Ceram. Soc. Bull. 51(4), 372 (1972).

18. S.M. Wiederhorn and H. Johnson: Effect of the electrolyte $\mathrm{pH}$ on crack propagation in glass. J. Am. Ceram. Soc. 56(4), 192 (1973). 